\title{
Atmospheric Neutrino Observations and Flavor Changing Interactions
}

\author{
M. C. Gonzalez-Garcia, ${ }^{1}$ M. M. Guzzo, ${ }^{2}$ P. I. Krastev, ${ }^{3}$ H. Nunokawa, ${ }^{2}$ O. L. G. Peres, ${ }^{1}$ V. Pleitez, ${ }^{4}$ \\ J.W.F. Valle, ${ }^{1}$ and R. Zukanovich Funchal ${ }^{5}$ \\ ${ }^{1}$ Instituto de Física Corpuscular-C.S.I.C., Departamento de Física Teòrica, Universitat of València, \\ 46100 Burjassot, València, Spain \\ ${ }^{2}$ Instituto de Física Gleb Wataghin, Universidade Estadual de Campinas, UNICAMP, 13083-970-Campinas, Brazil \\ ${ }^{3}$ University of Wisconsin, Madison, Wisconsin 53706 \\ ${ }^{4}$ Instituto de Física Teórica, Universidade Estadual Paulista, R. Pamplona 145, 01405-900 São Paulo, Brazil \\ ${ }^{5}$ Instituto de Física, Universidade de São Paulo, C.P. 66.318, 05389-970 São Paulo, Brazil
}

(Received 2 November 1998)

\begin{abstract}
Flavor changing (FC) neutrino-matter interactions can account for the zenith-angle-dependent deficit of atmospheric neutrinos observed in the SuperKamiokande experiment, without directly invoking either neutrino mass or mixing. We find that FC $\nu_{\mu}$-matter interactions provide a good fit to the observed zenith angle distributions, comparable in quality to the neutrino oscillation hypothesis. The required FC interactions arise naturally in many attractive extensions of the standard model. [S0031-9007(99)08969-3]
\end{abstract}

PACS numbers: 14.60.Pq, 14.60.St, 25.30.Pt, 96.40.Tv

Neutrinos produced as decay products in hadronic showers from cosmic ray collisions with nuclei in the upper atmosphere [1] have been observed by several detectors [2-7]. Although the absolute fluxes of atmospheric neutrinos are largely uncertain, the expected ratio $(\mu / e)$ of the muon neutrino flux $\left(\nu_{\mu}+\bar{\nu}_{\mu}\right)$ over the electron neutrino flux $\left(\nu_{e}+\bar{\nu}_{e}\right)$ is robust, since it largely cancels out the uncertainties associated with the absolute flux. In fact, this ratio has been calculated [1] with an uncertainty of less than 5\% over energies varying from 0.1 to $100 \mathrm{GeV}$. In this resides our confidence in the longstanding atmospheric neutrino anomaly.

Although the first iron-calorimeter detectors in Fréjus [2] and NUSEX [3] reported a value of the double ratio, $R(\mu / e)=(\mu / e)_{\mathrm{data}} /(\mu / e)_{\mathrm{MC}}$, consistent with one, all of the water Cherenkov detectors, Kamiokande [4], IMB [5], and SuperKamiokande [6], have measured $R(\mu / e)$ significantly smaller than one. Moreover, not long ago, the Soudan-2 Collaboration, also using an iron calorimeter, reported a small value of $R(\mu / e)$ [7], showing that the so-called atmospheric neutrino anomaly was not a feature of water Cherenkov detectors.

Recent SuperKamiokande high statistics observations [6] indicate that the deficit in the total ratio $R(\mu / e)$ is due to the number of neutrinos arriving in the detector at large zenith angles. Although $e$-like events do not present any compelling evidence of a zenith angle dependence, the $\mu$-like event rates are substantially suppressed at large zenith angles.

The $\nu_{\mu} \rightarrow \nu_{\tau}[6,8]$, as well as the $\nu_{\mu} \rightarrow \nu_{s}[8,9]$, oscillation hypothesis provides an appealing explanation for this smaller-than-expected ratio, as they are simple and well motivated theoretically. This led the SuperKamiokande Collaboration to conclude that their data provide good evidence for neutrino oscillations and neutrino masses.
In this Letter we give an alternative explanation of the atmospheric neutrino data in terms of flavor changing (FC) neutrino-matter interactions [10-14]. We show that, even if neutrinos have vanishing masses and/or the vacuum mixing angle is negligible, FC neutrino-matter interactions can still explain the SuperKamiokande data.

There are attractive theories beyond the standard model (SM), where neutrinos are naturally massless [15] as a result of a protecting symmetry, such as $B-L$ in the case of supersymmetric SU(5) models [16] and the model proposed in [17], or chiral symmetry in theories with an extended gauge structure, such as $\mathrm{SU}(3)_{c} \otimes \mathrm{SU}(3)_{L} \otimes$ $\mathrm{U}(1)_{N}$ (331)models [18]. The simplest example of this mechanism was first noted in an $\mathrm{SU}(2) \times \mathrm{U}(1)$ model proposed in Refs. [12,17], where a singlet Dirac lepton at the $\mathrm{TeV}$ scale is added sequentially to the SM in such a way that neutrinos remain massless due to an imposed $B$ - $L$ symmetry. The flavor mixing among the massless neutrinos in the leptonic charged current cannot be rotated away despite neutrinos being massless.

In general, models of neutrino mass are a natural source of FC neutrino-matter interactions. Seesaw-type models of neutrino mass have nondiagonal neutral current couplings of the $Z$ to mass eigenstate neutrinos [19] that may lead to new FC neutrino-matter interactions. Models of the radiative generation of neutrino mass [20] typically contain additional FC neutrino-matter interactions from scalar exchanges. Examples of this class are the supersymmetric models with broken $R$-parity [21]. Models with extended gauge structure, such as $E(6)$ models [15], may also lead to FC neutrino-matter interactions.

Here we focus on a massless neutrino conversion scenario as an explanation of the atmospheric neutrino data based on FC neutrino-matter interactions which induce $\nu_{\mu} \rightarrow \nu_{\tau}$ transition. Our results can be extended to the massive neutrino case. However, we stress that the 
present atmospheric neutrino data do not necessarily provide evidence for neutrino mass. Moreover, the existence of attractive theories, where FC neutrino-matter interactions do not imply neutrino mass, makes this possibility especially elegant. From a phenomenological point of view, FC interactions of neutrinos can induce flavor transitions when neutrinos travel through matter [10] irrespective of neutrino mass. In both massive [22] and massless $[12,13]$ cases, conversions can be resonant; however, the properties of the conversion are totally different. Massless neutrino conversions would be energy independent and would affect atmospheric neutrinos as well as antineutrinos, converting $\nu_{\mu} \rightarrow \nu_{\tau}$ together with $\bar{\nu}_{\mu} \rightarrow \bar{\nu}_{\tau}$. Remarkably, the present double ratio data do not show significant energy dependence $[6,23]$.

The presence of FC neutrino-matter interactions implies a nontrivial structure of the neutrino evolution Hamiltonian in matter. The evolution equations describing the $\nu_{\mu} \rightarrow \nu_{\tau}$ transitions in matter are given as $[12,13]$

$$
i \frac{d}{d r}\left(\begin{array}{c}
\nu_{\mu} \\
\nu_{\tau}
\end{array}\right)=\sqrt{2} G_{F}\left(\begin{array}{cc}
0 & \epsilon_{\nu} n_{f}(r) \\
\epsilon_{\nu} n_{f}(r) & \epsilon_{\nu}^{\prime} n_{f}(r)
\end{array}\right)\left(\begin{array}{c}
\nu_{\mu} \\
\nu_{\tau}
\end{array}\right) \text {, }
$$

where $\nu_{a} \equiv \nu_{a}(r), a=\mu, \tau$ are the probability amplitudes to find these neutrinos at a distance $r$ from their creation position, $\sqrt{2} G_{F} n_{f}(r) \epsilon_{\nu}$ is the $\nu_{\mu}+f \rightarrow \nu_{\tau}+f$ forward scattering amplitude, and $\sqrt{2} G_{F} n_{f}(r) \epsilon_{\nu}^{\prime}$ is the difference between the $\nu_{\tau}-f$ and $\nu_{\mu}-f$ elastic forward scattering amplitudes, with $n_{f}(r)$ being the number density of the fermions that induces such processes.

The use of the FC $\nu_{e}$-matter interactions was previously suggested in connection with the solar neutrino problem $[13,14]$. Recently, an attempt was made [24] to extend this idea in order to also account for the atmospheric neutrino data, but the fit obtained in this paper is not as good as our atmospheric neutrino fit or the solar fit in Ref. [14]. Moreover, some of the results in Table III of Ref. [24] seem inconsistent. On the other hand, Ref. [25] includes exotic flavor conserving $\nu_{\tau}$ interactions plus neutrino masses in order to account for the atmospheric and Liquid Scintillation Neutrino Detector (LSND) data, but without a detailed fit. We have decided to postpone the detailed analysis (within the present scenario) of the LSND, as well as of the solar neutrino data, for a future paper. Here we show that FC $\nu_{\mu}$-matter interactions can explain the atmospheric neutrino zenith angle anomaly, without introducing neutrino masses and/or mixing.

For our phenomenological approach let us simply assume the existence of a tree-level process $\nu_{\alpha}+f \rightarrow$ $\nu_{\beta}+f$ with amplitude proportional to $g_{\alpha f} g_{\beta f} / 4 m^{2}$, where $\alpha$ and $\beta$ are flavor indices, $f$ represents the interacting elementary fermion (charged lepton, $d$-like or $u$-like quark), and $g_{\alpha f}$ is the coupling involved in the vertex, where a $\nu_{\alpha}$ interacts with $f$ through a scalar or vector boson of mass $m$. The evolution equations which describe the $\nu_{\mu} \rightarrow \nu_{\tau}$ transitions in matter may be written generically, as in Eq. (1), where

$$
\epsilon_{\nu}^{\prime}=\frac{\left|g_{\tau f}\right|^{2}-\left|g_{\mu f}\right|^{2}}{4 m^{2} \sqrt{2} G_{F}} \quad \text { and } \quad \epsilon_{\nu}=\frac{g_{\tau f} \cdot g_{\mu f}}{4 m^{2} \sqrt{2} G_{F}} .
$$

Note also that, in the absence of neutrino mass, as we are assuming, antineutrino transitions $\bar{\nu}_{\mu} \rightarrow \bar{\nu}_{\tau}$ are governed by precisely the same evolution matrix in Eq. (1). We have calculated the transition probabilities of $\nu_{\mu} \rightarrow \nu_{\tau}\left(\bar{\nu}_{\mu} \rightarrow \bar{\nu}_{\tau}\right)$ and $P\left(\epsilon_{\nu}, \epsilon_{\nu}^{\prime}\right)\left[P\left(\epsilon_{\bar{\nu}}, \epsilon_{\bar{\nu}}^{\prime}\right)\right]$ as a function of the zenith angle by numerically solving the evolution equation using the density distribution in [26] and a realistic chemical composition with a proton/ neutron ratio 0.497 in the mantle and 0.468 in the core [27]. For the sake of simplicity we have assumed that $\epsilon \equiv \epsilon_{\bar{\nu}}=\epsilon_{\nu}$ and $\epsilon^{\prime} \equiv \epsilon_{\bar{\nu}}^{\prime}=\epsilon_{\nu}^{\prime}$, so there are only two free parameters in the analysis. We have used these probabilities to compute the theoretically expected number of $\mu$ - and $e$-like events $\left(N_{\mu}\right.$ and $\left.N_{e}\right)$ as a function of the two parameters, $\epsilon$ and $\epsilon^{\prime}$, for each of the five zenith angle bins for both the sub-GeV and the multiGeV SuperKamiokande data. Following Refs. [8,28], we fit separately the $\mu$ - and $e$-like events, taking into account the correlation of errors. The calculated numbers of events, $N_{\mu}$ and $N_{e}$, as functions of zenith angle, have been compared with the 535-day SuperKamiokande data sample in order to determine the allowed regions of $\epsilon$ and $\epsilon^{\prime}$ from a $\chi^{2}$ fit. We set our normalization by assuming that the relevant neutrino interaction in the Earth is only with down-type quarks. Any other scenario can be obtained from our results by rescaling the $\epsilon$ parameters.

In Fig. 1 we present a contour plot of the regions allowed by the SuperKamiokande data. The contour plots (a), (b), and (c) correspond to the regions allowed by the sub-GeV, multi-GeV, and combined SuperKamiokande data, respectively. These contours are determined by the

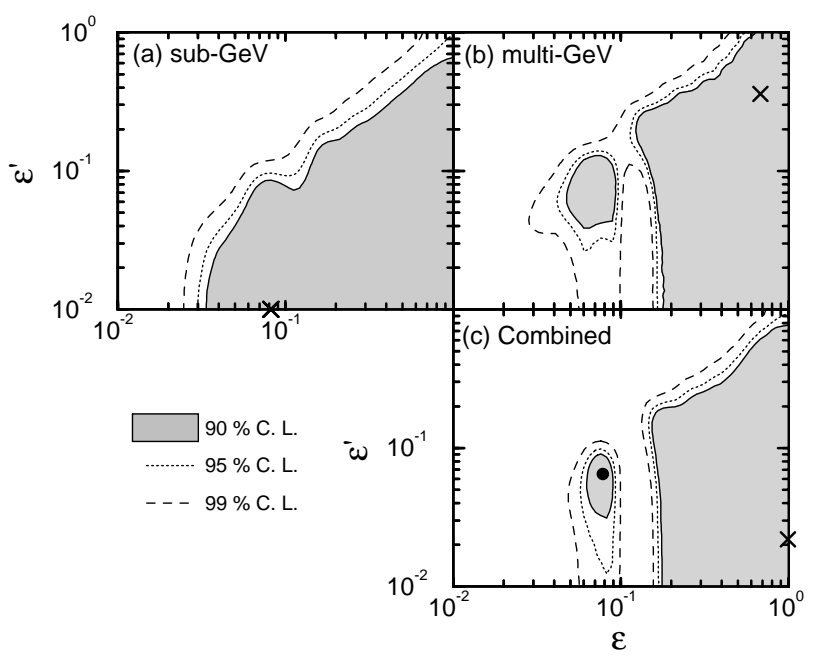

FIG. 1. Allowed region for $\epsilon$ and $\epsilon^{\prime}$ for SuperKamiokande (a) sub-GeV, (b) multi-GeV, and (c) combined events in the massless-neutrino scenario. The best fit points for each case are indicated by the crosses. 
conditions $\chi^{2}=\chi_{\min }^{2}+\Delta \chi^{2}$, where $\Delta \chi^{2}=4.6,6.0$, and 9.2 for $90 \%, 95 \%$, and $99 \%$ C.L., respectively.

In the parameter region that we have considered, i.e., $\epsilon$ and $\epsilon^{\prime}$ in the interval $[0.01,1.0]$, we found that $\chi_{\min }^{2}=$ 6.3 and 6.4 for the sub-GeV and multi-GeV samples, respectively ( 8 d.o.f. corresponding to ten data points minus two free parameters). These minima are obtained for $\left(\epsilon, \epsilon^{\prime}\right)=(0.08,0.01)$ and $(0.68,0.36)$, respectively, as indicated by the crosses in Fig. 1. For the combined case, $\chi_{\min }^{2}=14.7$ (18 d.o.f.) for $\left(\epsilon, \epsilon^{\prime}\right)=(0.99,0.02)$. In the combined case, the local best fit point $\left[\chi^{2}=16.9\right.$ for $\left.\left(\epsilon, \epsilon^{\prime}\right)=(0.08,0.07)\right]$ in the "island" determined by the 90\% C.L. curve is also indicated by a filled circle. This point is interesting because it still gives a good fit to the data with a relatively small value for the FC parameter $\epsilon$. We also find that the $\chi^{2}$ is relatively flat along the $\epsilon^{\prime}$ axis around the best fit point. The allowed regions can be qualitatively understood in the approximation of constant matter density. The conversion probability in this case is

$$
P\left(\nu_{\mu} \rightarrow \nu_{\tau}\right)=\frac{4 \epsilon^{2}}{4 \epsilon^{2}+\epsilon^{\prime 2}} \sin ^{2}\left(\frac{1}{2} \eta L\right),
$$

where $\eta=\sqrt{4 \epsilon^{2}+\epsilon^{\prime 2}} \sqrt{2} G_{F} n_{f}$. For $n_{f}=n_{d} \approx 3 n_{e}$ and $\epsilon^{\prime}<\epsilon$, the oscillation length in matter is given by

$$
L_{\mathrm{osc}}=\frac{2 \pi}{\eta} \approx 1.2 \times 10^{3}\left[\frac{2 \mathrm{~mol} / \mathrm{cm}^{3}}{n_{e}}\right]\left[\frac{1}{\epsilon}\right] \mathrm{km} .
$$

From Eq. (3), one can see that in order to have a large transition probability one must be in the region $\epsilon^{\prime} \lesssim \epsilon$ and $\eta \lesssim \pi / R_{\oplus}$. This last condition leads to a lower bound on $\epsilon$. The island in Fig. 1(b) corresponds to $\eta \sim \pi / R_{\oplus}$.

In Fig. 2 we give the expected zenith angle distribution of $\mu$-like sub-GeV events (a) and multi-GeV events (b) evaluated with our Monte Carlo program for the best fit points determined above. Our results clearly indicate an excellent fit for the $\mu$-like events, showing that they are highly depleted at $\cos \theta=-1$ with respect to the SM prediction. Note that, except for the assumption that the FC $\nu_{\mu}$-matter interaction involves $d$ quarks, our result is quite general, since we have not explicitly considered any particular model as the origin of the FC neutrino-matter interaction. Note that $e$-like events are not affected by the $\nu_{\mu} \rightarrow \nu_{\tau}$ transition.

What can we say about the required strength of the neutrino-matter interaction in order to obtain a good fit of the observed data? From our results and Eq. (2), we see that for masses $m \approx 200 \mathrm{GeV}$ we need at least $g_{\tau f}$. $g_{\mu f} \sim 0.1$ for the mixing term $\epsilon$. Similarly, our best fit $\epsilon^{\prime}$ value implies $\left|g_{\tau f}\right|^{2}-\left|g_{\mu f}\right|^{2} \sim 0.1$. While these values are relatively large, they are both weak-strength couplings. Moreover, they are consistent with present experimental bounds, for example, from universality of the weak interaction which is manifestly violated by Eq. (1).

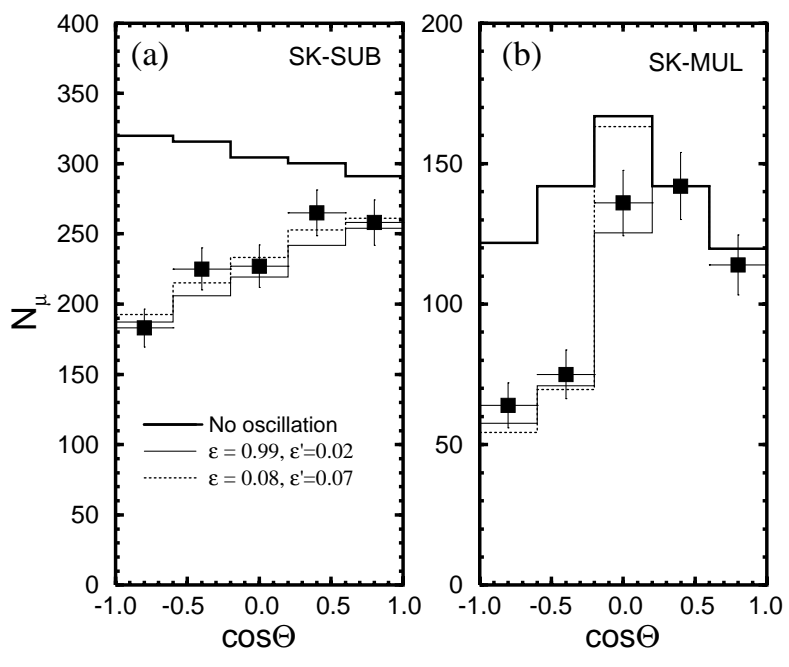

FIG. 2. Best fit zenith angle distributions in the masslessneutrino FC scenario (thin-solid and dotted lines) versus the nooscillation hypothesis (thick-solid line). The SuperKamiokande data are indicated by the crosses.

For the purpose of illustrating this explicitly, let us consider for the moment the supersymmetric model with broken $R$-parity [21] as a way to parametrize the FC neutrino-matter interaction. In this case, the FC $\nu_{\mu^{-}}$ matter interactions are mediated by a scalar down-type quark, $\tilde{d}_{j}$, so that we need only to check the couplings where a $d$ quark and a $\mu$ or $\tau$ neutrino are involved, i.e., $g_{i d} \approx \lambda_{i j 1}^{\prime}, i=2,3$. $\lambda_{i j k}^{\prime}$ are the coupling constants in the broken $R$-parity superpotential $\lambda_{i j k}^{\prime} L_{i} Q_{j} D_{k}^{c}$, where $L, Q$, and $D$ are standard superfields, and $4 \sqrt{2} G_{F} \epsilon=$ $\left|\sum_{j} \lambda_{3 j 1}^{\prime} \lambda_{2 j 1}^{\prime} / \tilde{m}_{\tilde{d} j}^{2}\right|$.

Constraints on the magnitude of such FC interactions in broken $R$-parity models have been given in [29]. The precision tests imply that individually these couplings are not severely constrained. The most stringent limit to the values of the relevant FC quantities comes from limits on the FC $\tau$ decay $B\left(\tau^{-} \rightarrow \rho^{0}+\mu^{-}\right)<6.3 \times 10^{-6}$ [30] which implies that $\left|\sum_{j} \lambda_{3 j 1}^{\prime} \lambda_{2 j 1}^{\prime}\left(100 \mathrm{GeV} / \tilde{m}_{\tilde{u} j}\right)^{2}\right|<$ $3.1 \times 10^{-3}$. Although a certain degree of fine-tuning is needed in order to verify this constraint, we find that the required strength of $\mathrm{FC} \nu_{\mu}$-matter interaction is consistent with all present data. One should note that broken $R$-parity supersymmetric models typically lead to neutrino masses which could be large. However, one may suppress them via fine-tuning or some additional symmetry.

In summary, we have demonstrated that flavor changing $\nu_{\mu}$-matter interactions can account for the zenithdependent deficit of atmospheric neutrinos observed in the SuperKamiokande experiment, without directly invoking neutrino masses and mixing. It provides a fit of the observations which is significantly better than the nooscillation hypothesis and is of a similar quality as the usual $\nu_{\mu} \rightarrow \nu_{\tau}$ oscillation hypothesis. The required FC interaction can arise in many attractive extensions of the $\mathrm{SM}$ and is consistent with all present constraints. 
The above FC mechanism can also be tested at future long baseline experiments. From Eq. (3), using $n_{e} \sim$ $2 \mathrm{~mol} / \mathrm{cm}^{3}, \epsilon \sim 1(0.1)$, and $\epsilon^{\prime}<\epsilon$, for the planned $\mathrm{K} 2 \mathrm{~K}$ experiment [31], one gets $P\left(\nu_{\mu} \rightarrow \nu_{\tau}\right) \sim 0.4$ (0.004) while, for MINOS [32], one finds $P\left(\nu_{\mu} \rightarrow \nu_{\tau}\right) \sim 0.9(0.2)$.

The existence of a massless neutrino explanation of the atmospheric neutrino anomaly may play an important theoretical role in model building, especially if one wants to account for all other hints for nonstandard neutrino properties, namely, the solar neutrino data, the LSND result, and the possible role of neutrinos as dark matter in the Universe [15].

We are grateful to M. Drees, Y. Grossman and Y. Nir for useful comments. This work was supported by the Spanish DGICYT under Grant No. PB95-1077, by the European Union TMR network ERBFMRXCT960090, by NSF Grant No. PHY96-05140, and by the Brazilian funding agencies CNPq, FAPESP, and the PRONEX program.

[1] L. V. Volkova, Sov. J. Nucl. Phys. 31, 784 (1980); M. Honda et al., Phys. Rev. D 52, 4985 (1995); V. Agrawal et al., ibid. 53, 1314 (1996); T. K. Gaisser and T. Stanev, ibid. 57, 1977 (1998).

[2] K. Daum et al., Z. Phys. C 66, 417 (1995).

[3] M. Aglietta et al., Europhys. Lett. 8, 611 (1989).

[4] K.S. Hirata et al., Phys. Lett. B 280, 146 (1992); Y. Fukuda et al., ibid. 335, 237 (1994).

[5] R. Becker-Szendy et al., Phys. Rev. D 46, 3720 (1992).

[6] Y. Fukuda et al., Phys. Lett. B 433, 9 (1998); Phys. Rev. Lett. 81, 1562 (1998); Phys. Lett. B 436, 33 (1998).

[7] W. W. M. Allison et al., Phys. Lett. B 391, 491 (1997); H. Gallagher et al., in Proceedings of the XXIX International Conference on High Energy Physics, Vancouver, Canada, 1998 (to be published).

[8] M. C. Gonzalez-Garcia, H. Nunokawa, O. L. G. Peres, T. Stanev, and J. W.F. Valle, Phys. Rev. D 58, 033004 (1998); M. C. Gonzalez-Garcia et al., hep-ph/9807305, Nucl. Phys. B (to be published).

[9] R. Foot, R. R. Volkas, and O. Yasuda, Phys. Rev. D 58, 013006 (1998); O. Yasuda, hep-ph/9809206.

[10] L. Wolfenstein, Phys. Rev. D 17, 2369 (1978); ibid. 20, 2634 (1979).

[11] M. Fukugita and T. Yanagida, Phys. Lett. B 206, 93 (1988).

[12] J. W. F. Valle, Phys. Lett. B 199, 432 (1987).
[13] M. M. Guzzo, A. Masiero, and S. Petcov, Phys. Lett. B 260, 154 (1991); E. Roulet, Phys. Rev. D 44, 935 (1991).

[14] V. Barger, R. J. N. Phillips, and K. Whisnant, Phys. Rev. D 44, 1629 (1991); P. I. Krastev and J. N. Bahcall, hep-ph/ 9703267; S. Bergman, Nucl. Phys. B515, 363 (1998).

[15] For a review, see J. W.F. Valle, hep-ph/9809234; Prog. Part. Nucl. Phys. 26, 91 (1991).

[16] L. J. Hall, V.A. Kostelecky, and S. Raby, Nucl. Phys. B267, 415 (1986); Y. Okada, hep-ph/9809297.

[17] R. Mohapatra and J. W.F. Valle, Phys. Rev. D 34, 1642 (1986); D. Wyler and L. Wolfenstein, Nucl. Phys. B218, 205 (1983).

[18] F. Pisano and V. Pleitez, Phys. Rev. D 46, 410 (1992); P. Frampton, Phys. Rev. Lett. 69, 2889 (1992).

[19] J. Schechter and J. W. F. Valle, Phys. Rev. D 22, 2227 (1980).

[20] A. Zee, Phys. Lett. 93B, 389 (1980); K. S. Babu, Phys. Lett. B 203, 132 (1988); for a review, see Ref. [15].

[21] L. Hall and M. Suzuki, Nucl. Phys. B231, 419 (1984); G. G. Ross and J.W.F. Valle, Phys. Lett. 151B, 375 (1985); J. Ellis, et al., ibid. 150B, 142 (1985); A. Masiero and J.W.F. Valle, Phys. Lett. B 251, 273 (1990); J. Romão, A. Ioannissyan, and J. W.F. Valle, Phys. Rev. D 55, 427 (1997).

[22] M. Mikheyev and A. Smirnov, Sov. J. Nucl. Phys. 42, 913 (1986).

[23] Some energy dependence observed in up-going muon events in SuperKamiokande could lead to additional restrictions to the FC solution to the atmospheric neutrino anomaly [see P. Lipari and M. Lusignoli, hep-ph/ 9901350].

[24] G. Brooijmans, hep-ph/9808498.

[25] E. Ma and P. Roy, Phys. Rev. Lett. 80, 4637 (1998).

[26] A. M. Dziewonski and D. L. Anderson, Phys. Earth Planet. Int. 25, 207 (1981).

[27] J. Bahcall and P. Krastev, Phys. Rev. C 56, 2839 (1997).

[28] G. Fogli and E. Lisi, Phys. Rev. D 52, 2775 (1995); G. Fogli, E. Lisi, D. Montanino, and G. Scioscia, ibid. 55, 485 (1997).

[29] V. Barger, G. F. Giudice, and T. Han, Phys. Rev. D 40, 2987 (1989); G. Bhattacharyya, Nucl. Phys. (Proc. Suppl.) A52, 83 (1997).

[30] C. Caso et al., Eur. Phys. J. C 3, 1 (1998).

[31] C. Yanagisawa et al., in Physics Beyond the Standard Model: From Theory to Experiment, edited by I. Antoniadis, L.E. Ibanez, and J.W.F. Valle (World Scientific, Singapore, 1998).

[32] D. Michael et al., Nucl. Phys. (Proc. Suppl.) B66, 432 (1998). 